\title{
ANNA M. BADIA MARTÍ: ASISTENCIA HUMANITARIA. REFLEXIONES DESDE LA PERSPECTIVA JURÍDICA INTERNACIONAL DE LOS PRINCIPIOS HUMANITARIOS Y ÉTICOS ${ }^{1}$.
}

\section{HUMANITARIAN ASSISTANCE, REFLECTIONS FROM THE INTERNATIONAL LEGAL VIEW OF THE ETHICAL AND HUMANITARIAN PRINCIPLE}

RESUMEN: Los ataques sistemáticos a la población civil y a las personas que prestan asistencia sanitaria son una constante en los conflictos armados actuales. Esta realidad se contrasta con los esfuerzos que se realizan para fortalecer los Principios Humanitarios - Humanidad, Imparcialidad, neutralidad

Esta contradicción es el objeto de estudio en este trabajo que se estructura en torno a tres apartados: 1. Fundamento de los Principios humanitarios en el Derecho Internacional de los Derechos Humanos, y el Derecho Internacional Humanitario; 2 El proclamado liderazgo del Secretario General de las NNUU en la coordinación de la asistencia humanitaria; 3 . La elaboración de un código ético de asistencia humanitaria

ABSTRACT: systematic attacks on the civilian population and persons providing health care are constant in today's armed conflicts. This reality contrasts with the ongoing efforts to strengthen humanitarian principles Humanity, Impartiality, Neutrality.

This contradiction is the object of study in this work, which is structured according to three sections: 1 . Ground of the humanitarian principles in the International Human Rights Law and in the International Humanitarian Law; 2. The proclaimed leadership of the Secretary General of the UN in the coordination of humanitarian assistance; 3 . The development of an ethical code of humanitarian assistance.

PALABRAS CLAVE: Códigos éticos, derecho internacional humanitario, derecho internacional de los derechos humanos, asistencia humanitaria, Principios humanitarios.

KEY WORDS: Ethical code, International Human Right Law, International Humanitarian Law, humanitarian assistance, Humanitarian principles.

\footnotetext{
${ }^{1}$ Catedrática de Derecho Internacional Público de la Universitat de Barcelona.
} 


\section{PLANTEAMIENTO.}

El punto de partida de nuestra reflexión se encuentra en el Derecho a la Salud, derecho humano internacionalmente reconocido ${ }^{2}$ y que por su propia naturaleza está intrínsecamente relacionado con el disfrute de otros derechos humanos: derecho a la alimentación, al trabajo, a la educación, a la vivienda, a la vida, a la no discriminación, a no ser sometido a torturas... . Se trata de un derecho humano, que desde la perspectiva de su exigibilidad se incluye en la categoría de Derechos económicos, sociales y culturales.

A partir de aquí, la cuestión que se plantea es su ejercicio en situación de conflicto armado u otra situación de emergencia, lo cual pone en marcha el concepto de "Prestación de asistencia sanitaria", formulado como un derecho a recibirla y una obligación de prestarla.

Además a ello ha de sumarse el tema general del Congreso: Ciencia y bioética nuevos retos compartidos, lo que por mi parte delimita mis reflexiones en torno a las cuestiones que considero relevantes y se tratan en los foros internacionales respecto a la necesidad de reafirmar los principios humanitarios, en una situación de violación reiterada de la prestación de asistencia humanitaria.

Según datos de la Cumbre Humanitaria mundial de Estambul de mayo de 2016, 125 millones de personas de todo el mundo necesitan asistencia humanitaria, hay 60 millones de desplazados y el Impacto del cambio climático evidencia un incremento de la frecuencia y gravedad de los desastres naturales. Además de los conflicto de amplio alcance de Siria, Iraq, Yemen, Sudan del Sur y Palestina, se contabilizan 35 conflictos activos.

Teniendo en cuenta las acotaciones hechas en torno a la exposición, me referiré a tres cuestiones, que a mi juicio son de interés respecto al tema asignado y que ponen de manifiesto, como cuestión de fondo, la permanente contradicción en que está sumergida la Sociedad internacional en la que coexisten comportamientos de barbarie en los conflictos armados y la adopción de medidas para hacer efectivos los principios humanitarios.

Me referiré con este orden a:

1. Fundamento de los Principios humanitarios en el Derecho Internacional de los Derechos Humanos y el Derecho Internacional Humanitario; en definitiva se trata de expresar la vigencia de los principios en que se sustenta la asistencia humanitaria como derecho a recibirla y obligación

\footnotetext{
2 Artículo 25 párrf. 1 de la Declaración universal de los derechos humanos, Art. 12 del Pacto Internacional de los derechos Economicos Sociales y Culturales.
} 
de prestarla, en toda situación de desastre, tanto por causas naturales como por intervención del hombre.

2 El proclamado liderazgo del Secretario General de las NNUU en la coordinación de la asistencia humanitaria. Me referiré al encargo que asumieron las NNUU en 1991 a través de una Resolución de la Asamblea General y asigno al Secretario General, de llevar a cabo la coordinación de la prestación de asistencia especialmente en casos de desastres naturales y sin perjuicio de la acción del Comité Internacional de la Cruz Roja (CICR). Asunción de responsabilidades que ha intentado revitalizar esta primavera pasado Ban ki-Moon a través de la Cumbre mundial Humanitaria celebrada en Estambul el 23 y 24 de mayo de 2016.

3. La elaboración de un código ético de asistencia humanitaria. Pone en evidencia la conveniencia de recordar que no es suficiente en este tema la aplicación de las normas jurídicas sino que ha de acudirse a la responsabilidad de todas las personas directamente implicadas en la necesidad de comportamientos éticos y moralmente aceptables. El código ético al que me refiero es el denominado "Principios éticos de la asistencia a la salud en tiempos de conflictos armados y otras situaciones de emergencia" aprobado el 30 de junio de 2015 bajo el paraguas del el $\mathrm{CICR}^{3}$ y se retoma la idea de implicar a toda la Comunidad Internacional, en la reciente iniciativa de la Asamblea General "Imperativos éticos para un mundo libre de armas nucleares"4.

\section{FUNDAMENTO DE LOS PRINCIPIOS HUMANITARIOS EN EL DERECHO INTERNACIONAL DE LOS DERECHOS HUMANOS $Y$ EL DERECHO INTERNACIONAL HUMANITARIO.}

Asegurar la asistencia sanitaria a toda persona que lo precise en situación de conflicto armado, es el objetivo central del Derecho Internacional humanitario (DIH), y lograr su efectividad, es la razón de ser del CICR.

Al referente de la situación de conflicto armado debe de añadirse las "situaciones de emergencia", que engloba: las calamidades provocadas por la naturaleza --terremotos, ciclones, erupción de volcanes...--; así como a las calamidades que se comenten por descontrol tecnológico --fugas químicas 0 radioactivas, rotura de presas...--- y como no, las calamidades causadas por el hombre, las derivadas de gobiernos hostiles y/o fracciones armada.

\footnotetext{
${ }^{3}$ Véase la "Carta de fecha 15 de octubre dirigida al Secretario General por el Representante Permanente de España ante las Naciones Unidas". Doc. S/2015/794, de 19 de octubre de 2015.

${ }^{4}$ A/RES/70/50 de 7 de diciembre de 2015.
} 
Los retos a los que se enfrenta el $\mathrm{DIH}$, en concreto en la clara preocupación por la dificultad en separar la asistencia humanitaria de los métodos de combate. Con frecuencia la prestación de asistencia, sea el personal que lo presta o los bienes de carácter sanitario, se convierten en objetivo de las partes en conflicto $y$, por tanto, en objetivo de la contienda bélica.

Esta realidad, que se viene documentando, entre otros, por el programa "Asistencia de salud en peligro $(\mathrm{HCID})^{5}$, rompe con dos de los pilares de la construcción del $\mathrm{DIH}$, que requiere por un lado, distinguir entre objetivo militar y objetivo civil y por otro no causar sufrimientos innecesarios a las víctimas del conflicto, sea combatiente o no.

La cuestión de fondo que se plantea, se deriva de la formalización del $\mathrm{DIH}$, y en concreto en la forma de hacerlo efectivo, ello nos sitúa ante los mecanismos de aplicación del Derecho internacional. El avance del DIH y su desarrollo se ha producido a medida que se modifican las técnicas y métodos de combate, convirtiéndose estos, en los impulsores del desarrollo normativo. En el momento actual, ha de sumarse el desarrollo de los derechos humanos, que nos dotan de un mínimo común denominador en los que se sustenta la prestación de asistencia humanitaria en todo tiempo y lugar. La concurrencia entre el DIH y el derecho internacional de los derechos humanos, debería conducir a la ausencia de vacíos legales, cuando se tratar de prestar asistencia humanitaria.

La fórmula de la que se parte es la siguiente: se aplicará el DIH en tiempo de guerra y el derecho internacional de los derechos humanos en tiempo de paz. Probablemente existe unanimidad en esta proposición, el problema surge en el momento de determinar cuál es el derecho aplicable a una situación de hecho y, en el momento de asegurar la garantía y eficacia, de los principios humanitarios mínimos ${ }^{6}$. Por ello se aboga por la formulación de unos Principios humanitarios que eviten cualquier interpretación que pueda conducir a una alteración de lo que se pretende, sea en tiempos de guerra 0 en tiempos de paz.

Principios humanitarios, que han de facilitar superar la formalización del Estado como parte del conflicto, y poder exigir el cumplimiento de las normas humanitarias a: "grupos", empresas ${ }^{7}$, organizaciones no-gubernamentales o a

\footnotetext{
${ }^{5}$ Véase el informe Health care in danger - violent incidents affecting the delivery of health care Jannuary 2012 to decembre 2014, ICRC, ref.4237.

${ }^{6}$ Principios que se concretan en el artículo 3 común a los cuatro Convenios de Ginebra de 1949, también conocida como cláusula Martens.

${ }^{7}$ Es relevante la participación de las empresas en los conflictos armados, tal como se pone de manifiesto en el Documento de trabajo del Centro Superior de Estudios de la Defensa Nacional Las corporaciones privadas de seguridad, No. 13/2015.
} 
particulares, en resumen a todo aquel que participa o pueda calificarse de parte en el conflicto.

La realidad de la pérdida de la centralidad del Estado, se evidencia en toda su crudeza en el campo del DIH, (tanto si lo contemplamos desde el prisma de las partes en conflicto como de los que prestan la asistencia humanitaria), ya que debemos hacerlo efectivo a través de los compromisos que asume el Estado.

En este ámbito es relevante la pertinaz insistencia, preocupándose por el tema de la aplicación del $\mathrm{DIH}$, y de protección de las personas que prestan la asistencia humanitaria por parte de la Asamblea General y del Consejo de Seguridad de las Naciones Unidas.

Muestra de esta afirmación es que la Asamblea General incluye en el programa de sus períodos de sesiones dos temas vinculados con nuestro trabajo: Seguridad del personal de la asistencia humanitaria y protección del personal de las Naciones Unidas ${ }^{8}$ y Situación de los Protocolos Adicionales de los convenios de Ginebra de 1949 relativos a la protección de las víctimas de los conflictos armados?.

El Consejo de Seguridad mantiene en su programa el tema "Protección de los civiles en los conflictos armados", lo que propicia la confección de los correspondientes informes del Secretario General sobre el tema. Junto a ello, ha de tenerse en cuenta los recordatorios pertinentes de aplicación del DIH en las controversias que figuran en su agenda.

Para completar el apoyo político diplomático a la aplicación del DIH, ha de señalarse la especial relevancia de la participación de los niños en los conflictos armados, también objeto del temario del Consejo de Seguridad y sobre el que se ha nombrado un Secretario General adjunto para velar por su aplicación ${ }^{10}$.

\section{EL PROCLAMADO LIDERAZGO DEL SECRETARIO GENERAL DE LAS NNUU EN LA COORDINACIÓN DE LA ASISTENCIA HUMANITARIA.}

Bajo este enunciado quiero llamar la atención de dos actividades que han recaído en la Secretaria de las NNUU. La primera es la referente a la asunción de las responsabilidades de Coordinación de la prestación de la asistencia humanitaria en las situaciones de emergencia; la segunda en el

\footnotetext{
${ }^{8}$.A/RES/69/133 de 12 de diciembre de 2014 (bianual).

${ }^{9} \mathrm{~A} / \mathrm{RES} / 69 / 120$ de 12 de diciembre de 2014 (bianual).

${ }^{10}$. Vid la Oficina del Representante Especial del Secretario General para la cuestión de los niños y los conflictos armados en https://childrenandarmedconflict.un.org/es/
} 
empeño del Sr. Ban Ki-Moon en convocar una Cumbre Humanitaria en mayo de 2016, acción de distinta naturaleza a la anterior pero que indica, como más adelante referiremos, los esfuerzos de la Secretaria en la materia que nos ocupa. Prestar la asistencia humanitaria forma parte del tercer propósito de la Organización: fomento de la Cooperación internacional en materia económica social, cultura y humanitaria, por tanto NNUU debe ejercitar y desarrollar sus competencias en asegurar la cooperación humanitaria.

\section{a) Coordinación de Naciones Unidas de la Asistencia Humanitaria emergencia (A/Res/46/182 de 19 de diciembre de 1991).}

Las resoluciones que fundamenta la actividad de coordinación en la prestación de la asistencia humanitaria, por parte de Naciones Unidas y encargado en concreto a la Secretaria son una Resolución de 19 de diciembre de 1991 y otra del 14 de diciembre de 1993 con el mismo título: "Fortalecimiento de la coordinación de la asistencia humanitaria de emergencia del sistema de Naciones Unidas" (A/RES/48/57).

En la primera se parte de unos Principios rectores, en concreto en tres. Primero los principios humanitarios (humanidad, neutralidad e imparcialidad), segundo, que se prestara la asistencia a petición del Estado que sufre la situación de emergencia y tercero se reitera que el Estado es el primer responsable.

El contenido de ambas resoluciones es organizar la competencia de las NNUU de prestar la asistencia humanitaria a quien lo solicite a través de por un lado dar cuerpo a la coordinación ${ }^{11}$ del conjunto de Estados, Organizaciones Internacionales intergubernamentales y No gubernamentales, programas y órganos --de las diferentes organizaciones universales y regionales--, incluye la gestión de las contribuciones voluntarias a través de un fondo que se articula para cada caso y, por otro lado liderar la coordinación de la prestación de la asistencia humanitaria, en la que se identifica cuatro fases; iniciación, organización, coordinación y prestación. Enfoque que significa afrontar la emergencia de la situación de hecho, la rehabilitación de los desastres causados y afrontar el desarrollo, de todo ello se encarga la Oficina de Coordinación de Asuntos Humanitarios ${ }^{12}$, con un Secretario General adjunto al frente.

La aprobación de estas Resoluciones no es ajena al contexto del debate en torna a: el derecho de asistencia humanitaria, la obligación de prestar la

\footnotetext{
${ }^{11}$ Es el Comité permanente entre organismo el que se ocupa de la coordinación (IASC, Inter-agency standing Committe)

${ }^{12}$ http://www.unocha.org/
} 
asistencia humanitaria y la controvertida intervención humanitaria ${ }^{13}$ y que en la actualidad, a partir de 2005, se trata bajo la noción de "responsabilidad de proteger" ${ }^{\prime \prime}$.

\section{b) Cumbre humanitaria.}

La Cumbre se realizó como se ha dicho a iniciativa del Secretario General, abundando en las competencias que se le habían atribuido en 1991 y 1993; pero además Ban Ki-Moon, quiere dar un impulso político-diplomático a la cuestión Humanitaria, de la misma forma que se habia convocado una cumbre para el desarrollo o la protección del medio ambiente, a ello se ve compelido por la gran cantidad de ataques dirigidos a los que prestan la asistencia y a la escasez de resultados frente a los medios empleados tanto humanos como materiales. Así como la mayor conciencia sobre los riesgos de catástrofes naturales.

El estudio de la cumbre, los tres años de consultas que la precedieron, los compromisos que se han alcanzado, y los cambios en la ayuda humanitaria, deben ser estudiados de forma monográfica, y realmente es muy sugerente. Aquí unas breves pinceladas en apoyo de la tarea realizada en el contexto de las NNUU, objeto de estas páginas.

La Cumbre se realizó en Estambul el 23 y 24 de mayo de 2016, se disponía de un documento de base elaborado por el Secretario General previas consultas $^{15}$. Resultados de la Cumbre son un llamamiento al cambio, estructurado en cinco líneas: Prevenir conflictos y ponerles fin, respetar las normas de guerra, no dejar nadie atrás, trabajar de manera diferente para poner fin a las necesidades e invertir en humanidad ${ }^{16}$. Líneas que cristalizan en una Agenda de humanidad a articular a través de una "Plataforma en acción" en la que se especificaran y darán publicidad a los Compromisos de los diferentes entes para la acción, adoptados en apoyo de la citada Agenda para la humanidad.

\footnotetext{
${ }^{13}$ Vid. Como referencia de la voluminosa bibliografia sobre la materia O.CORTEN et P.KEIN, Droit d'ingerencie ou obligation de reaction?, Editions Bruylant, 1992.

${ }^{14}$. Vid al respecto el reciente trabajo de C. GARCIA SEGURA y P.PAREJA ALCARAZ, "La responsabilidad cosmopolita de la responsabilidad de proteger: construcción normativa y disensos" en C.GARCIA SEGURA (dir) La tensión cosmopolita, avances y límites en la institucionalización del cosmopolitismo, Edit. Tecnos 2016 pp.64- 116

${ }^{15}$ Informe del Secretario General para la Cumbre humanitaria Mundial, Una Humanidad: nuestra responsabilidad compartida, Doc.A/70/709

${ }^{16}$. Vid. Doc A/71/353 “Resultados de la cumbre"
} 
A la espera de que se concluya la articulación de la Plataforma que está en curso, paulatinamente se va publicitando la información ${ }^{17}$. Señalar que el resultado de la Cumbre no ha sido acorde con las expectativas de su denominación. El término Cumbre se refiere a las convocatorias en las que la mayoría de asistentes son jefes de Estado y de gobierno, en este caso acudieron 55 jefes de Estados y de los considerados "grandes" sólo asistió Angela Merkel de Alemania. Por el lado de las organizaciones no gubernamentales, fue llamativa la postura de Médicos sin Fronteras, que se negaron asistir a un evento dirigido a recordar "las obligaciones de los Estados" ${ }^{\prime 18}$. Además como se ha indicado los compromisos están en curso, por tanto conviene continuar el seguimiento de la "Plataforma" y ver el grado de apoyo y valorar adecuadamente los cambios propuestos.

\section{LA ELABORACIÓN DE UN CÓDIGO ÉTICO DE ASISTENCIA HUMANITARIA.}

El 30 de junio de 2015 se aprobó, bajo el paraguas del Comité internacional de la Cruz Roja (CICR) un código ético titulado: "Principios éticos de la Asistencia a la Salud en Tiempos de Conflicto Armado y Otras situaciones de emergencia"19 ${ }^{\prime 1}$ título que indica su aplicación tanto a situaciones causadas por el hombre como de catástrofe natural.

Texto elaborado y aprobado por un conjunto de organizaciones y asociaciones de médicos y enfermeras directamente implicadas en la prestación de asistencia humanitaria. Acción que está amparada en la tradicional tarea que desempeña desde su creación el CICR y el Movimiento Internacional de la Cruz Roja y la Media Luna Roja y para este documento en concreto, el proyecto que viene desarrollándose desde 2011: Asistencia de Salud en peligro ${ }^{20}$.

\footnotetext{
17 Vid http://www.worldhumanitariansummit.org/

18 . Vid el interesesante articulo de C. NAUSSBAUM, "Cumbre humanitària Mundial, ¿'Y ahora que?, Estudios de Política Exterior, actualidad, de 27 de mayo de 2016.
}

Para un comentario de carácter más general VId M.J. IZQUIERDO ALBERCA, "I Cumbre Mundial Humanitaria de Estambul, necesidades y desafios globales", Instituto Español de Estuidios Estratégicos, Documento de Análisis, 47/2016.

19. Codigo reproducido en , "Carta de fecha 15 de octubre dirigida al Secretario General por el Representante Permanente de España ante las Naciones Unidas". Doc. S/2015/794, de 19 de octubre de 2015. Para un comentario sobre el mismo Vid A.BADIA, "Comentarios a los "Principios éticos de la asistencia a la salud en tiempos de conflictos armados y otras situaciones de emergencia", Comité Internacional de la Cruz Roja, 2015, en Revista Bioética y Derecho \&Perspectivas Bioéticas, 2016 no 36 pp. $125-133$

${ }^{20}$ www.healthcareindanger.org. 
Los autores del texto son cuatro organizaciones que agrupan diferentes profesionales: médicos, enfermeras y farmacéuticos, que prestan la asistencia humanitaria, además de ser respaldado por la "Federación Internacional de Asociaciones de Estudiantes de Medicina".

Tres de las organizaciones que han acordado el texto, son federaciones de profesionales, creadas en cada uno de los Estados de la nacionalidad de sus miembros de acuerdo con su ordenamiento interno, y con posterioridad se federan con asociaciones que tienen el mismo carácter. Todas ellas llevan una consolidada trayectoria. Desde la perspectiva jurídica internacional pueden calificarse de Organizaciones no-gubernamentales, de las que responden a asociaciones de profesionales, y participan en los procesos de formación de normas, y tienen un especial interés en su colaboración, por el área de trabajo, en el contexto de los Organismos especializados del Sistema de Naciones Unidas $^{21}$.

La cuarta organización, es de diferente naturaleza jurídica internacional, es una Organización internacional de carácter intergubernamental; se trata del Comité Internacional de Medicina Militar (CIMM-ICMM) $)^{22}$.Las cuatro organizaciones representan a más de 30 millones de profesionales sanitarios de los ámbitos civil y militar.

El texto no tiene naturaleza jurídica vinculante, generador de derecho y obligaciones o regulador de las relaciones mutuas, naturaleza que corresponde al conjunto normativo del DIH y del Derecho Internacional de los derechos humanos. Estamos ante un "código ético", dirigido a promover los principios éticos, identificados por las organizaciones autoras del mismo, entre sus miembros -- médicos, enfermeros y farmacéuticos--, y tiene el carácter complementario de generar pautas de conducta para potenciar la aplicación del marco jurídico normativo citado.

Es por ello que el código, entrando ya en el contenido, establece que el personal sanitario actuará de acuerdo con: i) el derecho internacional y nacional; ii) los principios éticos de asistencia a la salud y iii)de acuerdo con su conciencia individual. En base a ellos prestará en todo momento la mejor asistencia, teniendo en cuenta el uso equitativo de recursos; en consecuencia aplicando los tres principios: humanidad, --conforme el cual se ha de prevenir y

\footnotetext{
${ }^{21}$ Federación Farmacéutica Internacional (FFI) www.fip.org, que agrupa 132 organizaciones. La Asociación Médica Mundial (AMM) www.wma.net creada en 1947 en París, y que tiene en su haber ser autora de las Declaraciones de Helsiky, que constituye un código deontológico a nivel mundial sobre el ejercicio de la medicina. Consejo Internacional de Enfermería (CIE) www.icn.ch, que constituye una federación de más de 130 asociaciones de enfermería.

22 www.cimm-icmm.org. Creada el 21 de julio de 1921 y cuenta con más de 110 estados Miembros, España es Miembro originario.
} 
aliviar en todas las circunstancias el sufrimiento humano --imparcialidad y neutralidad --cuyo significado es prestar asistencia sin discriminación--. Principios totalmente consolidados en el Ordenamiento Jurídico Internacional y desarrollados en el ámbito del DIH desde que se incorporó esta materia en el mismo, a partir de la Convención para la mejora de la suerte de los heridos en campaña de 22 de agosto de 1864 y que es el origen del Movimiento Internacional de la Cruz Roja.

La función del personal de salud es proteger la salud física y mental y aliviar los sufrimientos, proporcionando los cuidados con humanidad, respetando la dignidad del paciente y sin discriminación. En consecuencia, no aceptará ni participara en actos de tortura.

El personal sanitario, goza de protección funcional para prestar la asistencia humanitaria; se les reconoce privilegios e inmunidades a través de los ordenamientos internos, protección de la que se verán desposeídos cuando se realicen otras actividades.

Las relaciones del paciente con el personal sanitario están presididos por los principios de la bioética: consentimiento informado del paciente, imparcialidad y no discriminación, y confidencialidad respeto a la intimidad del paciente, ampliamente consensuado en los códigos éticos sanitarios y cristalizados en la Declaración universal sobre "Bioética y Derechos Humanos" de la UNESCO de 19 de octubre de 2005.

Por último el código se refiere a la protección del personal que prestan la asistencia, así como el material y transporte que necesitan para realizar dicha asistencia. Las personas y el material deben estar perfectamente identificados con los símbolos internacionalmente protegidos, tal como se establece en los Convenios de Ginebra y sus protocolos ${ }^{23}$. Deberán, además, poder acceder a los pacientes y a las instalaciones. Asimismo se afirma que no podrán ser castigados por el cumplimiento de normas jurídicas o éticas.

En otro orden de ideas y en este caso relacionado con la carrera de armamentos, otra vertiente del que clásicamente se conocía como derecho de guerra, constatar que mayoritariamente se ha visto la conveniencia de incentivar los compromisos éticos en materia humanitaria cuando estamos ante armas nucleares. Efectivamente, a partir del 70 Aniversario de las NNUU se ha incluido dentro del tema: desarme general y completo tres sub-temas que vinculan armas nucleares y principios humanitarios y éticos, son: "Compromisos humanitarios para la prohibición y la eliminación de las armas

\footnotetext{
23. En concreto es ilustrativo el Protocolo III Adicional a los Convenios de Ginebra, relativo a la aprobación de un signo distintivo adicional, de 8 de diciembre de 2005 (BOE de 18 de febrero de 2011, No. 42).
} 
nucleares"24, "Consecuencias humanitarias de las armas nucleares" ${ }^{25}$ e " Imperativos éticos para un mundo libre de armas nucleares"26.

Se parte de la realidad de que de producirse la utilización de armas nucleares, ningún ente sería capaz de afrontar la ayuda humanitaria, por ello se considera que debe actuar en todos los frentes para lograr su prohibición y alcanzar un Tratado general y vinculante. Una forma de incentivarlo es alertar sobre las consecuencias que comportan para los principios humanitarios y la conveniencia de recordar los compromisos éticos que afectan a la comunidad en su conjunto.

Se insta "... a la comunidad internacional a no escatimar esfuerzos individuales y colectivos para promover el imperativo ético de un "concepto más amplio de libertad"', y se reconoce que "...los imperativos éticos para el desarme nuclear y la urgencia de lograr y mantener un mundo libre de armas nucleares, que es un "bien público mundial de primer orden" que responde a intereses tanto nacionales como de seguridad colectiva"27.

Resoluciones que se aprueban por mayoría de votos, pero no se alcanza el consenso ni la unanimidad ${ }^{28}$, se está en la confrontación entre la mayoría no nuclear $^{29}$ y la minoría poseedora de armas nucleares.

\section{CONSIDERACIONES FINALES.}

Sin ánimo de exhaustividad de los diferentes elementos apuntados a lo largo de estas páginas, creo oportuno hacer algunas concreciones respecto los puntos de partida que hemos planteado en la introducción. Me referiré sintéticamente a: 1 .Supuesto de hecho al que se dirige la prestación de asistencia humanitaria; 2. Prevención y continuidad en la prestación de asistencia humanitaria. 3. Revitalización de los principios humanitarios y éticos y su implicación a los diferentes actores de la Sociedad Internacional. 4. Renovación de la responsabilidad de los Estados en que se produce la emergencia humanitaria, 5. ¿Cabe distinguir entre códigos éticos y aplicación de Los Principios humanitarios a través del Derecho Internacional Humanitario y el Derecho internacional de los derechos humanos?

\footnotetext{
${ }^{24}$ A/RES/70/48, de 7 de diciembre de 2015

${ }^{25}$ A/RES/70/47, de7 de diciembre de 2015

${ }^{26}$ A/RES/70/50, de 7 de diciembre de 2015

${ }^{27}$ Ibid, preambulo y párrf 2

28 . Dependiendo de la resolución se contabilizan, entre 30 y 40 votos en contra y en torno a 20 abstenciones.

${ }^{29}$ El compromiso humanitario adoptado en la Conferencia de Desarme de 2015, fué suscrito por 114 Estados (Doc. CD/2039)
} 
1.La prestación de la asistencia humanitaria, en su formulación jurídica de derecho a recibirla y obligación de prestarla, y cuyo régimen jurídico deriva o del DIH o del DIDH, afecta del mismo modo a las situaciones de hecho provenientes de la intervención del hombre (guerras, disturbios, fracciones armadas, gobiernos hostiles, descontrol tecnológico de materias peligrosas...) como de la situación de emergencia derivada de las fuerzas de la naturaleza (terremotos, ciclones, erupción de volcanes...).

2.La prestación de la asistencia humanitaria, atendiéndonos a las precisiones realizadas en el marco de las NNUU a través de las tareas del Secretario General --liderar la competencia de cooperación en la asistencia humanitaria e iniciativa político-diplomática de la Cumbre humanitaria--, comprende tanto la prevención de las causas que conducen a la situación de emergencia --aquí también lo prueba las resoluciones que vinculan, armas nucleares y principios humanitarios y éticos--, dar respuesta a la emergencia una vez se ha producido y, continua con la reconstrucción, vinculándose a la cooperación al desarrollo. Concepción de la prestación que hoy por hoy no coincide con los medios materiales que se disponen, ni tampoco a los resultados de los medios invertidos.

3. Asistimos a una revitalización de los principios humanitarios -en la medida en que continúan y aumentan el número de víctimas de los que prestan la asistencia, junto con las víctimas de la catástrofe o emergencia propiamente dicha --. Sobre ellos cabe afirmar que coinciden su dimensión ética y jurídica, por ello los destinatarios de los mismos son: los Estados, las OOII, las ONGs, programas, empresas, grupos de ciudadanos e individuos particulares...., afectan por tanto a todos los participantes de la Comunidad Internacional. Ello, a la luz de los últimos desarrollos, permite afirmar la oportunidad de mantener las dos vertientes tanto de derecho positivo como de principios o códigos éticos, facilitando en definitiva la superación del papel central del Estado en la aplicación uniforme del Ordenamiento Jurídico Internacional.

4. Después de invertir cantidades importantes de dinero y causar bajas y menosprecio en el personal que suministra la asistencia, convirtiéndose en víctimas de la situación de emergencia. La tendencia es volver a potenciar la Responsabilidad de los Estados como primer implicado en el tema, y recordar que la solidaridad no sustituye la responsabilidad del que la tiene atribuida. Ello se manifiesta en la tendencia sobre los cambios que sustenta el Secretario General en la Cumbre humanitaria y conceptualmente nos sitúa en la categoría jurídica de la "Responsabilidad de proteger".

5. La respuesta a la cuestión planteada, si cabe distinguir entre códigos éticos por un lado y aplicación de los Principios humanitarios a través del 
Derecho Internacional Humanitario y el Derecho internacional de los derechos humanos por otro. A mi juicio, por lo que se refiere al contenido este coincide y debe hacerse todo lo necesario para su difusión en este sentido. Por lo que se refiere al mantenimiento de ambos, es decir la dimisión jurídica positiva y dimensión la ética, creo que deben mantenerse, por ser una de las formas ideneas para fomentar una aplicación homogénea y universal de los derechos humanos internacionalmente reconocidos, al mismo tiempo que facilita la implicación de todos los actores.

\section{BIBLIOGRAFIA CITADA:}

A.BADIA, "Comentarios a los "Principios éticos de la asistencia a la salud en tiempos de conflictos armados y otras situaciones de emergencia", Comité Internacional de la Cruz Roja, 2015, en Revista Bioética y Derecho \&Perspectivas Bioéticas, 2016 № 36 pp. 125-133.

Centro Superior de Estudios de la Defensa Nacional Las corporaciones privadas de seguridad, No. 13/2015.

COMITÉ INTERNACIONAL DE LA CRUZ ROJA (ICRC), Health care in danger - violent incidents affecting the delivery of health care - January 2012 to decembre 2014, ref.4237.

O.CORTEN et P.KEIN, Droit d'ingerence ou obligation de reaction?, Editions Bruylant, 1992.

C. GARCIA SEGURA y P.PAREJA ALCARAZ, "La responsabilidad cosmopolita de la responsabilidad de proteger: construcción normativa y disensos" en C.GARCIA SEGURA (dir) La tensión cosmopolita, avances y límites en la institucionalización del cosmopolitismo, Edit. Tecnos 2016 pp.64- 116

M.J. IZQUIERDO ALBERCA, "I Cumbre Mundial Humanitaria de Estambul, necesidades y desafíos globales", Instituto Español de Estuidios Estratégicos, Documento de Análisis, 47/2016.

C. NAUSSBAUM, "Cumbre humanitaria Mundial, ¿Y ahora qué?, Estudios de Política Exterior, actualidad, de 27 de mayo de 2016. 